\section{S. Obenauer}

S. Luftner-Nagel

D. von Heyden

U. Munzel

F. Baum

E. Grabbe

\title{
Screen film vs full-field digital mammography: image quality, detectability and characterization of lesions
}

Published online: 24 July 2002

(C) Springer-Verlag 2002
S. Obenauer $(\bowtie) \cdot$ S. Luftner-Nagel D. von Heyden · F. Baum · E. Grabbe Department of Radiology,

Georg-August-Universität Göttingen, Robert-Koch-Strasse 40,

37075 Göttingen, Germany

e-mail: soben@med.uni-goettingen.de Tel.: +49-551-398965

Fax: +49-551-399606

The online version of the original article can be found at

http://dx.doi.org/10.1007/s00330-001-

$1269-\mathrm{y}$
U. Munzel

Department of Medical Statistics,

Georg-August-Universität Göttingen,

37075 Göttingen, Germany
Robert-Koch-Strasse 40,

\section{Eur Radiol (2002) 12:1697-1702}

Throughout the article, "BI-RADS" TM" was incorrectly rendered as "BI-RADSZZZ". 